Model for laser ablation plume expansion in gas

This article has been downloaded from IOPscience. Please scroll down to see the full text article.

2005 J. Phys. D: Appl. Phys. 381892

(http://iopscience.iop.org/0022-3727/38/12/007)

View the table of contents for this issue, or go to the journal homepage for more

Download details:

IP Address: 161.111.22.141

The article was downloaded on 12/12/2012 at 11:15

Please note that terms and conditions apply. 


\title{
Model for laser ablation plume expansion in gas
}

\author{
Casper V Budtz-Jørgensen ${ }^{1,3}$, Michael M Mond ${ }^{2}$, \\ Brendan Doggett ${ }^{1}$ and James G Lunney ${ }^{1,4}$ \\ ${ }^{1}$ Physics Department, Trinity College, Dublin 2, Republic of Ireland \\ ${ }^{2}$ Department of Mechanical Engineering, Ben-Gurion University of the Negev, Beer Sheva, \\ Israel \\ E-mail: jlunney@tcd.ie
}

Received 12 November 2004, in final form 5 April 2005

Published 3 June 2005

Online at stacks.iop.org/JPhysD/38/1892

\begin{abstract}
A simple model for laser ablation plasma plume expansion in a background gas is presented. The expanding plume is approximated by a semi-ellipsoidal shell of infinitesimal thickness and with a mass corresponding to the amount of background gas that has been snowploughed by the shell as it expands. Furthermore, the internal pressure of the plume and external pressure of the background gas are taken into account. Electrical probe measurements of ns laser ablation of Ag were used to map out the angular dependence of plume expansion in argon at various pressures and the results compared with model predictions.
\end{abstract}

\section{Introduction}

Pulsed laser deposition (PLD) is well-established as a method for depositing novel thin films for research [1,2]. The technique relies on the ablation of a bulk material using a pulsed laser and subsequent condensation of the expelled material on a substrate, which is typically positioned some centimetres away from the target. Under vacuum conditions (below $10^{-4}$ mbar) the expansion dynamics of the ablation plume is relatively well understood and may be described by the self-similar model of Anisimov et al [3,4], which approximates the plume by a semi-ellipsoid that expands adiabatically in a self-similar way. For PLD of many materials it is necessary to use a gaseous atmosphere during deposition. The gas may react with the plume material or on the growing surface and be incorporated in the film. Furthermore, the interaction of the ablation plume with the gas may decrease the average energy of the ablated species impacting on the film, thus, for example, minimizing re-sputtering and other undesirable effects. A systematic experimental study of the temporal evolution of the ablation plume in a gas and how it differs from the vacuum case was first performed by Geohegan [5] using ICCD imaging. Ablation of YBCO in oxygen showed a relatively well-defined contact surface between the ablation plume and the ambient gas

\footnotetext{
3 Present address: Instituto de Optica, CSIC, Calle Serrano 121, 28006 Madrid, Spain.

4 Author to whom any correspondence should be addressed.
}

and its expansion perpendicular to the target surface could be qualitatively reasonably well described by the spherical blast wave model (i.e. a $R \propto t^{2 / 5}$ spherical expansion). Langmuir probes [6] and optical emission [7] spectroscopy have also been used to observe the forward expansion of the plume and the results fitted with the spherical blast wave model.

Although other theoretical [8,9] and numerical [10] models that do go beyond the spherical blast wave description have been proposed these do not try to, in a simple manner, describe the angular variation of plume expansion. Thus, the primary aim of the work reported here was to develop such a simple model of the expansion of a laser ablation plume in a gas that would take into account the non-spherical nature of the expansion. The contact surface itself is approximated by a semi-ellipsoid of infinitesimal thickness and with a mass corresponding to the amount of ambient gas that has been swept during expansion. Furthermore, the forces acting upon it due to the internal and external pressures are taken into account. The choice of the semi-ellipsoid is based on its success in describing the angular variation in expansion kinetics under vacuum conditions $[3,4,11]$. To test the model a series of electrical probe measurements of silver target laser ablation in vacuum and in an argon gas at various pressures were performed. The pressure regime was chosen so that plume splitting [12] was observed thus giving a clear indication of the location of the contact surface. The time-of-flight (TOF) of the contact surface at different angles with respect to the 


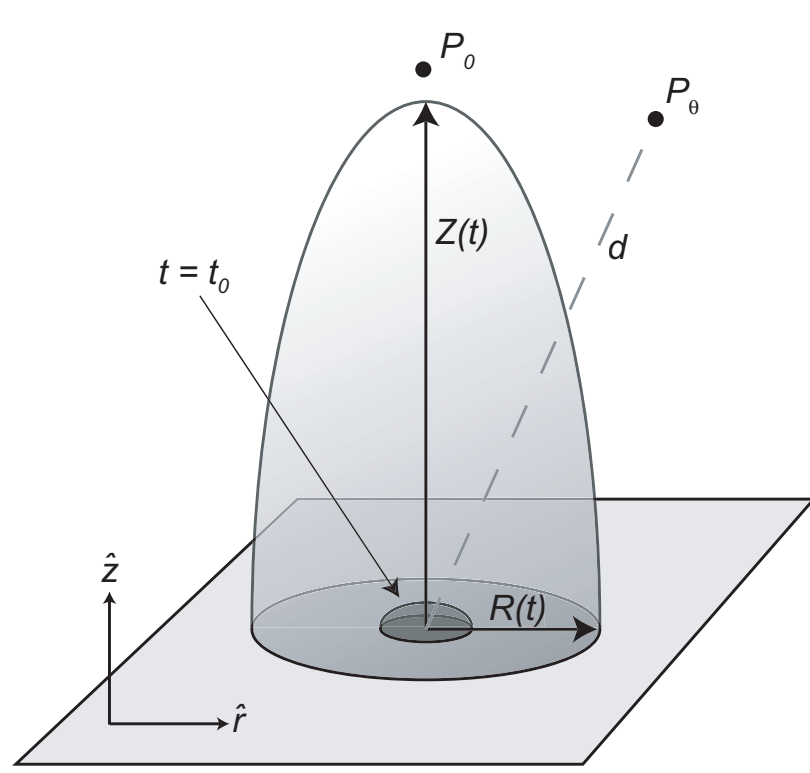

Figure 1. Schematic of the ellipsoidal geometry used in the model. A circular laser spot is assumed. $P_{0}$ and $P_{\theta}$ denote two different probe positions.

target normal was then mapped out and compared with the model.

\section{Theoretical model}

As mentioned, the expansion of a plume into vacuum has been investigated extensively both theoretically and experimentally. The self-similar model of Anisimov et al [3,4] describes the plume as an ellipsoid (see figure 1) that, shortly after the laser is turned off, expands freely into a vacuum in such a way that every volume element moves radially away from the centre with a constant velocity. Within the framework of that model, it is possible to obtain explicit expressions for the various physical variables that characterize the expansion and in particular the angular dependence of such quantities as the TOF as well as the thickness of the deposited film.

The picture changes drastically when the plume expands into an ambient gas, as the flow inside the plume becomes more complex as a result of its coupling to the flow in the neighbouring medium. Thus, in the first stage immediately after the beginning of the expansion, the contact surface between the plume and the ambient gas acts as a piston that drives an external shock wave into the ambient gas. The contact surface in its turn is slowed down (relative to the free expansion) due to the mass of the ambient gas that is accumulated behind the external shock wave. This slowdown of the contact surface gives rise to an internal shock wave in the plume that reduces the velocity of the freely expanding volume elements to that of the contact surface velocity. At early stages of the expansion the contact surface together with the two shock waves on both its sides constitute a thin layer whose velocity is close to the free expansion velocity. However, as the accumulated mass of the ambient gas becomes comparable to the mass of the plume, the internal shock wave moves inwards, reaches the centre and consequently bounces back and forth between the centre and the contact discontinuity. The dynamics of the plume is elucidated by considering the evolution of the relative magnitude of its kinetic and thermal energies. Initially, the plume's energy is mainly kinetic as it freely expands into the ambient gas. As the internal shock wave starts to move inwards, kinetic energy is converted into thermal energy due to the slowing down of increasing portions of the plume. Thus, when the internal shock wave reaches the centre most of the plume's kinetic energy is converted into thermal energy and, to a good approximation, the plume's density and temperature become uniform. Several of the stages of the dynamics of plume expansion described above have been investigated theoretically under the assumption of spherical symmetry (e.g. [13]). Such phenomena as the dynamics of supernovae remnants as well as plume expansion for PLD applications have been studied within the framework of such models. Spherically symmetric models are quite useful in gaining insight into the main processes that govern the dynamics of the plume expansion. However, when, as in laser plasma deposition applications, the angular dependence of the physical variables that describe the plasma is of importance, those models obviously cannot provide an adequate description. In order to overcome that difficulty, a model is developed here that takes into account the angular variation of the physical variables. The underlying assumption of the model is that the accumulated ambient mass forms a thin layer between the contact surface and the external shock wave. This assumption is quite plausible and is valid well after the internal shock wave detaches from the contact surface and reaches the centre. In addition, it is assumed that the thin layer is of the shape of a semi-ellipsoid surface of the form

$$
1-\frac{r^{2}}{R(t)^{2}}-\frac{z^{2}}{Z(t)^{2}}=0
$$

where $r$ and $z$ are the surface radial and axial coordinates, respectively, while $R(t)$ and $Z(t)$ are the minor and major axes of the ellipsoid, respectively. The axis origin is located at the centre of the laser spot and the axial direction is perpendicular to the target plane. The velocity of a mass element on the surface is given by the following:

$$
\boldsymbol{u}=\frac{r}{R(t)} \dot{R}(t) \hat{r}+\frac{z}{Z(t)} \dot{Z}(t) \hat{z}
$$

where $\hat{r}$ and $\hat{z}$ are unit vectors in the radial and axial directions, respectively. It should be noted that equations (1) and (2) are consistent with each other in the sense that the solution of the equation

$$
\frac{\mathrm{d} \boldsymbol{r}}{\mathrm{d} t}=\boldsymbol{u}(\boldsymbol{r})
$$

with $\boldsymbol{u}$ given by equation (2) is given by equation (1). Obviously, the aim now is to find the functions $R(t)$ and $Z(t)$ that govern the dynamics of the plume. In order to do that, the momentum equation of the entire layer is written and is given by

$$
\frac{\mathrm{d} \wp}{\mathrm{d} t}=\sum \boldsymbol{F},
$$

where $\wp$ is the total linear momentum of the accumulated mass layer and $\sum \boldsymbol{F}$ denotes the sum of all the forces acting on it. 
In order to express the total momentum of the layer in terms of $R(t)$ and $Z(t)$, it is written as

$$
\wp=\int_{A} \boldsymbol{u} \sigma \mathrm{d} a,
$$

where $\boldsymbol{u}$ is the velocity of the accumulated mass as given by equation (2), $\sigma$ is its surface mass density and the integration is over the ellipsoid surface. Generally, the surface mass density is a function of the location along the surface. However, calculating $\sigma$ is outside the scope of the current model, and instead the average velocity is defined as

$$
\overline{\boldsymbol{u}}=\frac{\int_{A} \boldsymbol{u} \sigma \mathrm{d} a}{\int_{A} \sigma \mathrm{d} a},
$$

where the denominator on the right-hand side of equation (6) is the total mass of the accumulated ambient gas, and is given by

$$
\int_{A} \sigma \mathrm{d} a=\frac{2 \pi}{3} R^{2}(t) Z(t) \rho_{\mathrm{s}}
$$

where $\rho_{\mathrm{s}}$ is the density of the undisturbed ambient gas. Equation (7) is based on the assumption that for probes that are situated far enough from the target the homogenization time scale of the plume is much shorter than the TOF while the thin layer assumption is still valid. Thus, $t=0$ signifies the beginning of the homogeneous plume stage at which time the mass of the accumulated ambient gas is equal to the plume's mass. Such a time interval is quite wide as is indicated by the numerical simulations of Arnold et al [13] of their spherical model, and includes the time scales of the TOF that characterize typical PLD conditions. In order to calculate the average velocity, equation (2) is employed together with the following definition:

$$
X=\frac{R}{Z},
$$

in order to yield the following expression:

$$
\overline{\boldsymbol{u}}=\dot{R}(t) \Gamma_{r}(X) \hat{r}+\dot{Z}(t) \Gamma_{z}(X) \hat{z},
$$

where

$$
\begin{aligned}
& \Gamma_{r}(X)=\frac{\int_{0}^{1} \eta^{2}(\xi) \sigma(\xi) \sqrt{1+X^{2} \eta^{\prime 2}(\xi)} \mathrm{d} \xi}{\int_{0}^{1} \eta(\xi) \sigma(\xi) \sqrt{1+X^{2} \eta^{\prime 2}(\xi)} \mathrm{d} \xi} \\
& \Gamma_{z}(X)=\frac{\int_{0}^{1} \xi \eta(\xi) \sigma(\xi) \sqrt{1+X^{2} \eta^{\prime 2}(\xi)} \mathrm{d} \xi}{\int_{0}^{1} \eta(\xi) \sigma(\xi) \sqrt{1+X^{2} \eta^{\prime 2}(\xi)} \mathrm{d} \xi}
\end{aligned}
$$

and

$$
\eta(\xi)=\sqrt{1-\xi^{2}} .
$$

Starting with the uniform surface mass distribution case, numerical calculations indicate that the dependence of $\Gamma_{r}$ and $\Gamma_{z}$ on $X$ is very weak and hence constant values $\left(\Gamma_{r 0}=0.8\right.$ and $\left.\Gamma_{z 0}=0.5\right)$ are employed for that case. Furthermore, when non-uniformity of the surface mass distribution is taken into account, the values of $\Gamma_{r}$ and $\Gamma_{z}$ become even less dependent on $X$ than the uniform case, and may be presented in the following form:

$$
\begin{aligned}
& \Gamma_{r}=f_{r} \Gamma_{r 0}, \\
& \Gamma_{z}=f_{z} \Gamma_{z 0},
\end{aligned}
$$

where $f_{r}$ and $f_{z}$ are correction factors due to non-uniformity of the surface mass distribution. Within the framework of the current model the factors $f_{r}$ and $f_{z}$ serve as free parameters in order to adjust the theoretical model to the experimental results. As will be seen later on, their values are quite close to unity.

Turning to the right-hand side of equation (4), the forces that act on the accumulated mass are due to the external as well as internal pressures. The external constant pressure is due to the undisturbed ambient gas and is denoted by $p_{\mathrm{g}}$. As a consequence of assuming that the plume is homogeneous, the internal pressure is spatially uniform but varies with time as the plume is expanding, according to the following equation

$$
p_{\mathrm{p}}(t)=(\gamma-1) E\left[\frac{2 \pi R^{2}(t) Z(t)}{3}\right]^{-1},
$$

where $E$ is the thermal energy of the plume, and $\gamma$ is the specific heat ratio of the plume.

The specific heat ratio, $\gamma$, is different during the two main stages of the expansion. During the free expansion the temperature is low enough so that the plasma is kinetically active, and hence the specific heat ratio has been justifiably taken by various researchers to be around 1.25. However, as the plume is stopped by a series of internal shock waves that propagate back and forth, most of its energy is converted into thermal energy and it heats up considerably. As a result, the plasma is expected to be highly ionized and $5 / 3$ is a more adequate value for the specific heat ratio during that stage.

The total force acting on the accumulated mass can be calculated now and is given by

$$
\sum F=\left(p_{\mathrm{p}}-p_{\mathrm{g}}\right) \int_{A} \hat{n} \mathrm{~d} a,
$$

where $\hat{n}$ is the unit vector normal to the ellipsoid surface given by equation (1). To summarize, the two components of the momentum equation are

$f_{r} \Gamma_{r 0} \frac{\mathrm{d}}{\mathrm{d} t}\left[\frac{2 \pi}{3} R^{2}(t) Z(t) \rho_{\mathrm{g}} \frac{\mathrm{d} R}{\mathrm{~d} t}\right]=\left(p_{\mathrm{p}}-p_{\mathrm{g}}\right) \int_{A} \hat{n}_{r} \mathrm{~d} a$,

$f_{z} \Gamma_{z 0} \frac{\mathrm{d}}{\mathrm{d} t}\left[\frac{2 \pi}{3} R^{2}(t) Z(t) \rho_{\mathrm{g}} \frac{\mathrm{d} Z}{\mathrm{~d} t}\right]=\left(p_{\mathrm{p}}-p_{\mathrm{g}}\right) \int_{A} \hat{n}_{z} \mathrm{~d} a$,

where it should be noted that the integrals on the right-hand sides are functions of $R(t)$ and $Z(t)$.

\section{Solutions}

The two coupled differential equations given by equations (17) and (18) may be solved numerically using a given set of initial conditions. During the initial stage of the expansion, when the plume mass and density are significantly larger than the accumulated mass and ambient gas density, respectively, free expansion can be assumed. Thus, the model of Anisimov et al remains valid during this stage. At some point the presence of the ambient gas will start to influence the expansion of the plume. It is this point in time and space we choose to start our 
model. Thus, defining $t=0$ as the time when the accumulated mass equals the plume mass, i.e.

$$
\frac{2 \pi}{3} R(0)^{2} Z(0) \rho_{\mathrm{g}} \equiv M_{\mathrm{p}}
$$

and solving for $R(0)$ and $Z(0)$, we get

$$
\begin{gathered}
R(0)=\left(\frac{3 M_{\mathrm{p}}}{2 \pi \rho_{\mathrm{g}} k_{0}}\right)^{1 / 3}, \\
Z(0)=k_{0} R(0),
\end{gathered}
$$

where we have used the definition $k_{0} \equiv Z(0) / R(0)$ for the plume elongation at $t=0$.

Inserting typical values $\left(M_{\mathrm{p}}=130 \mathrm{ng}, \rho_{\mathrm{g}}(10 \mathrm{~Pa} \mathrm{Ar})=\right.$ $1.78 \times 10^{-4} \mathrm{~kg} \mathrm{~m}^{-3}$ ) into equations (20) and (21) yields $R(0) \approx$ $0.5 \mathrm{~cm}$ and $Z(0) \approx 1.2 \mathrm{~cm}$ where we have used $k_{0}=2.4$ (see next section). The solutions to equations (17) and (18) are actually rather insensitive to the exact choice of $R(0)$ and $Z(0)$, especially at the later times which are of main interest to us here.

Numerical solutions to equations (17) and (18) using the the initial conditions given in table 1 are shown in figure 2 in terms of $R(t), Z(t)$ and the plume elongation $k=Z(t) / R(t)$ (figure 2, left) and in terms of $\dot{R}(t), \dot{Z}(t)$ and the normalized accumulated mass (figure 2, right). Also shown is the spherical expansion calculated using the expression for the blast wave model [14]:

$$
R_{\text {b.w. }}(t)=\varepsilon_{0}\left(\frac{2 E_{0}}{\rho_{\mathrm{g}}}\right)^{1 / 5} t^{2 / 5},
$$

where $\varepsilon_{0}=1.12$ for $\gamma=5 / 3$. The energy in equation (22) is multiplied by a factor of 2 so that the full spherical expansion

Table 1. Initial conditions derived from Ag target ablation measurements in vacuum using the Anisimov interpretation and equations (20) and (21).

\begin{tabular}{ll}
\hline$R(0)=0.5 \mathrm{~cm}$ & $M_{\mathrm{p}}=130 \mathrm{ng}$ \\
$Z(0)=1.2 \mathrm{~cm}$ & $\rho_{\mathrm{g}}=1.78 \times 10^{-4} \mathrm{~kg} \mathrm{~m}^{-3}$ \\
$\dot{R}(0)=21 \mathrm{~km} \mathrm{~s}^{-1}$ & $E_{0}=18 \mathrm{~mJ}$ \\
$\dot{Z}(0)=50 \mathrm{~km} \mathrm{~s}^{-1}$ & $k_{0}=2.4$ \\
\hline
\end{tabular}

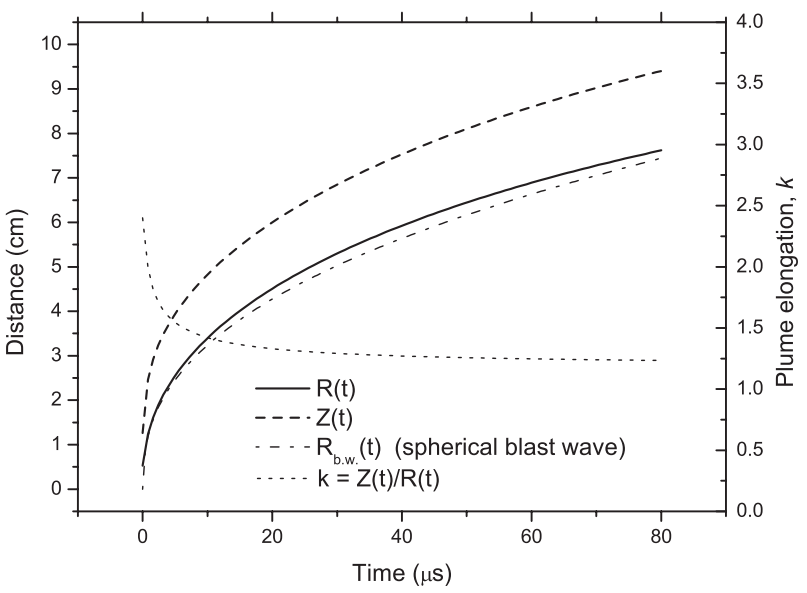

of the blast wave model can be directly compared to the semiellipsoidal expansion of the model presented here. It may be noted that solutions to the model using $k_{0}=1$ and $f_{r}=f_{z}=1$ (not shown) coincide with the blast wave model for both $R(t)$ and $Z(t)$. Thus, the solutions to the model presented here simplify to the spherical blast wave model upon full spherical symmetry.

From the plots it can be seen that the deceleration of the plume expansion which is also characteristic of the spherical blast wave model is seen for both the $R$ and $Z$ components of the expanding plume (left). Also, as can be seen from the plume elongation $k \equiv Z(t) / R(t)$, the background gas has the effect of making the plume more spherical with time. From the solutions to $\dot{R}(t)$ and $\dot{Z}(t)$ the rapid slowdown from free expansion at early times due to the accumulation of background gas can be clearly observed.

\section{Experiments and comparison}

To test the model against experiment a series of Langmuir probe measurements of plasmas created by pulsed laser ablation of silver targets in argon were performed. These were done for a number of different argon pressures. For each pressure, the ion signal was recorded at different angles with respect to the target normal. Furthermore, a set of measurements was done under vacuum conditions allowing us to determine the initial velocity components of the plume before plume-gas interactions become significant.

\subsection{Experiment}

A ns-pulsed $(10 \mathrm{~Hz}) 1064 \mathrm{~nm} \mathrm{Nd:YAG} \mathrm{laser} \mathrm{beam} \mathrm{was}$ directed at normal incidence onto a rotating silver target. The laser fluence was typically around $9 \mathrm{~J} \mathrm{~cm}^{-2}$ in a circular spot with a diameter of $1 \mathrm{~mm}$. The angular distribution of the ablated flux was measured using a flat ion probe with an area of $5.3 \mathrm{~mm}^{2}$ and a distance of $7.5 \mathrm{~cm}$ from the target for most of the measurements. Furthermore, the weight loss per pulse was measured by weighing the target before and after a large number of laser pulses and was found to be around $130 \mathrm{ng} / \mathrm{shot}$.

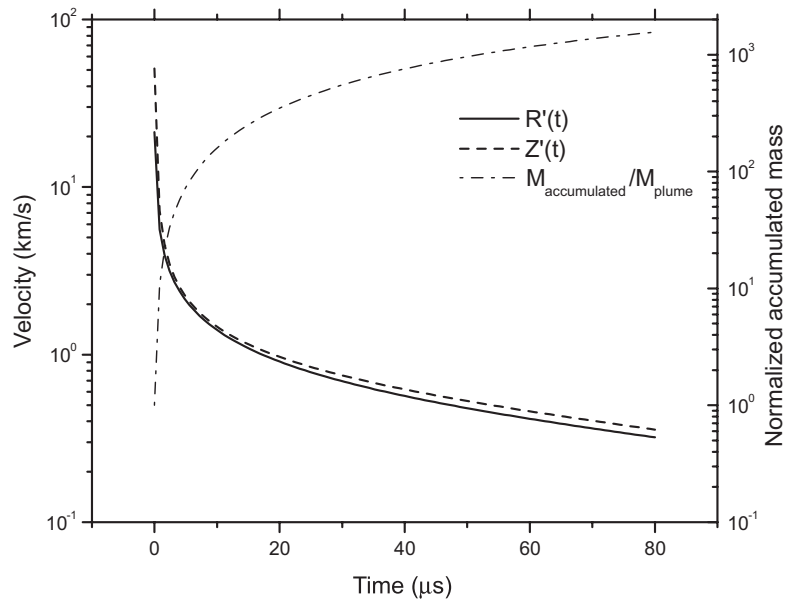

Figure 2. Left: Solutions for $R(t)$ and $Z(t)$ and the elongation of the plume, $k$. Also shown is the spherical blast wave expansion, $R_{\mathrm{b} . w .}(t)$, calculated for an energy of $2 E_{0}$. Right: Solutions for $\dot{R}(t)$ and $\dot{Z}(t)$ and the normalized accumulated mass. See table 1 for initial conditions. The correction factors used are $f_{z}=0.4$ and $f_{r}=0.6$ (see section 4.3). An argon gas pressure of 0.10 mbar was used. 

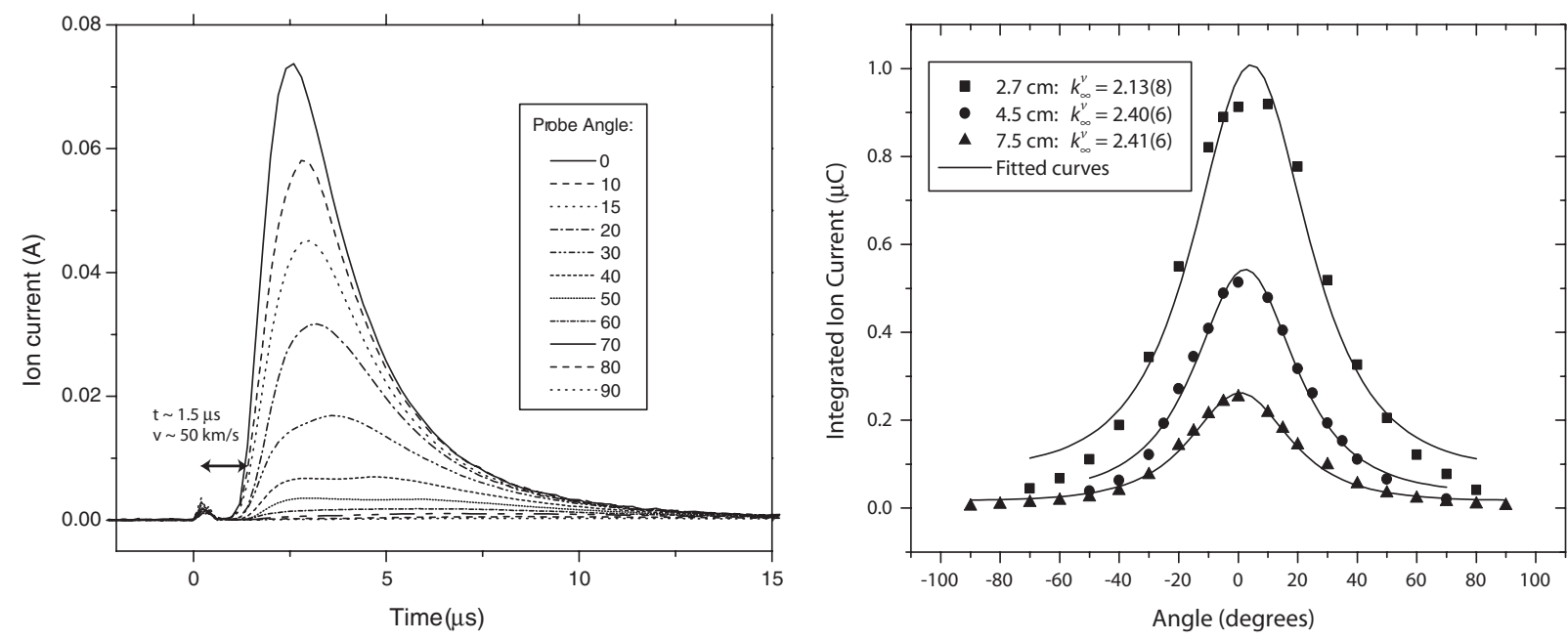

Figure 3. Left: Ion probe TOF scans for laser ablation of $\mathrm{Ag}$ in vacuum at a probe distance of $7.5 \mathrm{~cm}$. Traces for different probe angles between $0^{\circ}$ and $90^{\circ}$ are shown. Right: Integrated ion currents as a function of probe angle for 3 different probe distances (symbols) and fits of equation (23) to the data (curves). The plume elongation, $k_{\infty}^{v}$, extracted from the fits is shown in the legend.

\subsection{Measurements in vacuum}

As mentioned above, measurements performed in vacuum were used to determine the initial conditions of the ellipsoidal plume during the onset of plume-gas interaction. Since plume expansion in vacuum quickly reaches an asymptotic constant velocity, measurement of the velocity components and plume elongation, $k$, at typical probe distances of some centimetres may be used as a qualified estimate of these quantities also at earlier times closer to the target. Figure 3 (left) shows ionprobe signals measured at a distance of $7.5 \mathrm{~cm}$ from the target at varying probe angles (see inset in figure 4 for angle definition). The decrease in ion signal with probe angle is indicative of the forward directed expansion which is characteristic of ablation plumes. We use the model of Anisimov et al to parametrize the expansion at this point in terms of the asymptotic velocities $\dot{Z}_{\infty}^{v}$ and $\dot{R}_{\infty}^{v}$ and the asymptotic plume elongation $k_{\infty}^{v}$. By integrating the signals in figure 3 (left) and plotting as a function of probe angle we may fit the resulting distribution with the expression [3]:

$$
Q(\theta)=Q(0)\left[1+\left(k_{\infty}^{2}-1\right) \sin \theta\right]^{-3 / 2},
$$

which denotes the total integrated flux of ablated material expanding along an angle $\theta$. The distributions and fits calculated in this way are shown in figure 3 (right) for a number of probe distances together with the plume elongations, $k$, which may be extracted from the fits (see legend). An asymptotic plume elongation of $k_{\infty}^{v}=2.4$ for expansion in vacuum may thus be established. $\dot{Z}_{\infty}^{v}$ may be estimated directly from the ion signal at $0^{\circ}$ by noting the TOF of the plume front (see figure) yielding $\dot{Z}_{\infty}^{v}=50 \mathrm{~km} \mathrm{~s}^{-1}$. Assuming that we are well within the asymptotic expansion region, we may write $\dot{R}_{\infty}^{v} \sim \dot{Z}_{\infty}^{v} / k_{\infty}^{v}=21 \mathrm{~km} \mathrm{~s}^{-1}$. Knowing the velocity components and plume elongation of this ellipsoidal expansion, its total kinetic energy, $E_{\text {kin }}$, can be calculated using the following expression, which is easily derived from the Anisimov formalism (again assuming constant expansion):

$$
\left(\frac{\mathrm{d} Z}{\mathrm{~d} t}\right)^{2}=\frac{2(5 \gamma-3)}{(\gamma-1)\left(1+2 / k_{\infty}^{2}\right)} \frac{E_{\mathrm{kin}}}{M},
$$

where $M$ is the plume mass and we use a value of $\gamma=1.25$ [11] for the adiabatic constant yielding a value of $E_{\mathrm{kin}}=18 \mathrm{~mJ}$. As was explained before, the time it takes the plume to reach its asymptotic velocities is very small in comparison to the TOF in the experiment. In addition, the homogenization time is also small in comparison with the latter as may be learnt from the fast inward motion of the internal shock wave [13]. Hence, it is assumed that asymptotically, relative to the TOF, the asymptotic free expansion as well as the homogenization of the plume are both achieved at time $t=0$ and from then on most of the plume's energy is thermal.

Some caution must be exercised when using the model of Anisimov et al on ion probe measurements, since this model considers the plume to consist of neutrals, whereas ion probe measurements detect only the ionic part of the plume. Although the neutral and ionic parts of the plume might well be decoupled, the rather good fits achieved with this model in figure 3 (right) shows that it atleast serves well as a parametrization of the ionic part of the plume. In the present context, where the ion measurements are used to establish the initial conditions of a plume expanding in a gas, one would ideally rely on equivalent measurements of the neutral component, since the neutrals most likely constitute the bulk of the plume. Thus, in relying on ion probe measurements in the present case, we are assuming that the expansions of the ionic and neutral parts are not too dissimilar during the initial stage of the expansion.

\subsection{Measurements in argon}

Following the measurements in vacuum, equivalent measurements were performed in an argon gas using a probe distance of $7.5 \mathrm{~cm}$. The ion probe signals obtained in this way are shown in figure 4 (left) for an argon pressure of 0.10 mbar. Two distinct peaks are clearly visible: the fast peak is mainly due to the non-colliding fraction of the ablated material and a slow peak (indicated with an arrow) due to the shock front created by plume-gas interaction. Taking the TOF of the second peak as an indication of the plume-gas contact surface and plotting it 

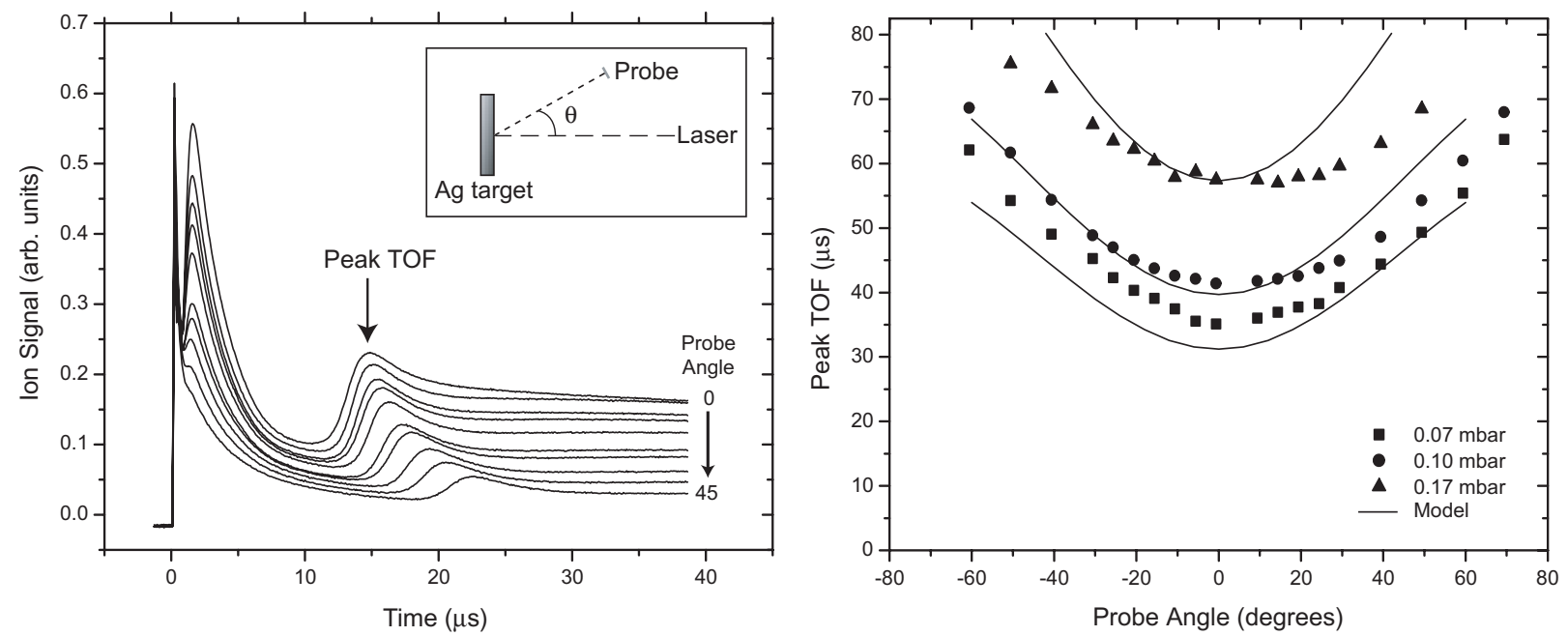

Figure 4. Left: Ion probe TOF scans for laser ablation of Ag in 0.10 mbar argon. Traces for different probe angles between $0^{\circ}$ and $45^{\circ}$ are shown. Right: Measured peak TOF values at various angles compared with the model.

as a function of probe angle, the resulting TOF distribution can be compared with the model. The resulting plots of the measured TOFs for different argon pressures are shown in figure 4 (right, symbols). Also shown are the calculated curves, which were based on $Z(t)$ and $R(t)$ for the initial conditions shown in table 1 and with the relevant argon pressure applied in their calculation. Furthermore, the correction factors $f_{z}=0.4$ and $f_{r}=0.6$ (see equations (13) and (14)) were chosen in a way so as to achieve best overall agreement between measurements and the model for the three pressures.

\section{Discussion}

From the experimental TOF curves shown in figure 4 it is clear that a spherically symmetric model, such as the blast wave model, is not capable of fully describing the the dynamics of plume expansion in a background gas. Indeed, within such a model no angular variation of the peak TOF, as seen in figure 4 (right), can be achieved. The present model allows for such an angular dependence confined within an ellipsoidal symmetry, while retaining the temporal expansion of the blast wave model which has previously [5] been shown to adequately describe plume expansion in the forward direction. The reason for the similarity between the models resides in the fact that both rely on the sweeping of accumulated background gas by the initial release of energy. However, in contrast to the blast wave model, which assumes the energy release to be that of a point explosion, our model takes into account the inhomogeneity of the energy release. This inhomogeneity is parametrized in terms of the model of Anisimov et al by fitting their model to measurements in vacuum, and subsequently using the resulting fit parameters (i.e. the asymptotic velocities and plume elongation) as the initial conditions for our model. The underlying assumption here is that the initial stages of expansion both in vacuum and in a background gas will be similar. This approach is feasible if the onset of attenuated expansion (i.e. $t=0$ ) takes place after the asymptotic velocities of the equivalent expansion in vacuum has been reached. Evaluation of the model of Anisimov et al shows this to be the case.
The comparison made in figure 4 (right) shows good overall agreement between the experimental data and the theoretical model both as a function of probe angle and background gas pressure. Although some fitting of the model to the experimental data has taken place through the choice of $f_{z}$ and $f_{r}$, these factors were kept at a constant for all the theoretical curves. It is, however, not obvious that the distribution of accumulated mass, and thus $f_{z}$ and $f_{r}$, would be the same at different pressures and, hence, further fine tuning of the model could be performed by adopting pressure dependent correction factors. However, for the purpose of testing the overall efficiency of the model against the present experiments constant correction factors were chosen.

\section{Conclusion}

A simple theoretical model describing laser ablation plasma plume expansion in the presence of a background gas has been presented. The model approximates the plume as a thin ellipsoidal shell with total mass corresponding to the amount of accumulated background material expanding under the influence of the internal and external forces acting upon it. Electrical probe measurements were used to map out the angular dependence of the contact surface between the expanding plume and background gas at different argon gas pressures. Although some correction parameters describing the inhomogeneity of the accumulated mass were used as fitting parameters good agreement with the experimental data was seen both as a function of probe angle and argon gas pressure.

\section{Acknowledgments}

This work was supported by the EU Growth Programme under contract G6RD-CT1999-0084 and by the Irish Research Council for Science, Engineering and Technology.

\section{References}

[1] Chrisey D B and Hubler G K (ed) 1994 Pulsed Laser Deposition of Thin Films (New York: Wiley) 
[2] Miller J C and Haglund Jr R F (ed) 1998 Laser Ablation and Absorption (New York: Academic)

[3] Anisimov S I, Bauerle D and Luk'yanchuk B S 1993 Phys. Rev. B 4812076

[4] Anisimov S I, Luk'yanchuk B S and Luches A 1996 Appl. Surf. Sci. 96-98 24

[5] Geohegan D B 1992 Appl. Phys. Lett. 602732

[6] Hansen T N, Schou J and Lunney J G 1999 Appl. Surf. Sci. 138-139 184

[7] Amoruso S, Bruzzese R, Spinelli N, Velotta R, Wang X and Ferdeghini C 2002 Appl. Phys. Lett. 804315

[8] Baranov V Yu, Derkach O N, Grishina V G, Kanevskii M F and Serbant A Yu 1993 Phys. Rev. E 481324
[9] Bulgakov M and Bulgakova N M 1998 J. Phys. D: Appl. Phys. 31693

[10] Itina T E, Hermann J, Delaporte P and Sentis M 2002 Phys. Rev. E 66066406

[11] Hansen T N, Schou J and Lunney J G 1999 Appl. Phys. A 69 601

[12] Wood R F, Chen K R, Leboeuf J N, Puretzky A A and Geohegan D B 1997 Phys. Rev. Lett. 791571

[13] Arnold N, Gruber J and Heitz J 1999 Appl. Phys. A 6987

[14] Zel'dovich Ya B and Raizer Yu P 2002 Physics of Shock Waves and High-Temperature Hydrodynamic Phenomena (New York: Dover) 


\section{Corrigendum}

Model for laser ablation plume expansion in gas

C V Butz-Jørgensen, M M Mond, B Doggett and J G

Lunney 2005 J. Phys. D: Appl. Phys. 38 1892-1898

The caption for figure 4 on page 1897 should be changed to read as:

Figure 4. Left: Ion probe TOF signals at $4.5 \mathrm{~cm}$ from the target and at various angles for laser ablation of $\mathrm{Ag}$ in $0.12 \mathrm{mbar}$ of argon. Right: Angular distribution of the TOF values for the second peak maximum recorded at $7.5 \mathrm{~cm}$ from the target in various pressures of argon compared with the theoretical model. 